\title{
Presenting the Area of a Right Triangle as the Sum of an Infinite Series
}

\author{
Salman Mahmud* \\ Student of BIAM Model School And College, Bogura Bangladesh.
}

*Corresponding Author: Salman Mahmud, Student of BIAM Model School And College , Bogura Bangladesh.

It is very easy to calculate the area of a triangle. If we ask anyone who has a little knowledge of mathematics, he will say that it is $\frac{1}{2} \times$ basexheight or he will mention the other formulas to calculate the area of a triangle. Here in this article I will present the area of a right triangle as the sum of an infinite series (convergent). From that infinite series we can also get the formula $\frac{1}{2} \times$ basexheight. Believe it or not, mathematics is the most beautiful subject because mathematics can represent anything in different interesting ways.

\section{The square inscribed in a right triangle}

Before we present the area of a right triangle as the sum of an infinite series, we need a formula which will help us to calculate the length of one side of a square inscribed in a right triangle so that one vertex of the square coincides with right-angle vertex of the right triangle.

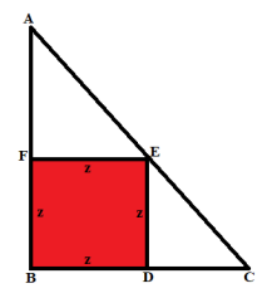

Figure1. $B D E F$ is a square inscribed in the right triangle $A B C$.

Suppose, $z$ is the length of one side of the square $B D E F$. We can see, right triangle $A E F$ and right triangle $C D E$ are similar. That's why,

$\frac{A F}{Z}=\frac{Z}{D C} \Rightarrow \frac{A B-Z}{Z}=\frac{Z}{B C-Z} \Rightarrow Z^{2}-Z^{2}+A B \cdot Z+B C \cdot Z=A B \cdot B C$

$\Rightarrow Z(A B+B C)=A B \cdot B C \quad \Rightarrow Z=\frac{A B \cdot B C}{A B+B C}$

So this is the formula to calculate the length of one side of a square inscribed in a right triangle.

\section{Presenting the area of a right triangle as the sum of an infinite series}

To present the area of a right triangle as the sum of an infinite series, here we will use an interesting method. So firstly look at the figure below,

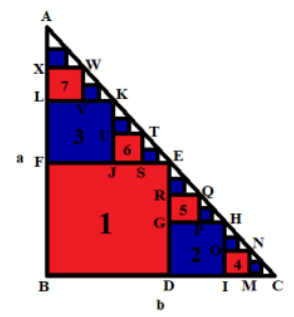

Figure2. $A B C$ is the given right triangle. 
In the figure 2, firstly I have drawn the square $B D E F$ (square 1) inscribed in the triangle $A B C$. For this we have got two new right triangles $\triangle C D E$ and $\triangle A E F$. Then I have drawn two squares $D G H I$ (square 2) and $F J K L$ (square 3) inscribed in the triangles $\triangle C D E$ and $\triangle A E F$ respectively. Again, I have got four more right triangles $\triangle C H I, \triangle E G H, \triangle K E J$ and $\triangle A K L$. Then similarly I have drawn four squares (square 4,5,6,7) inscribed in those four right triangles. For this I have got eight more right triangles. If WE continue this process up to infinity, we will get infinite number of squares. If we add the areas of those squares, we will get the area of the triangle. But it is totally impossible to calculate the areas of all squares one by one up to infinity and add them. So if we get a pattern at the time of adding the areas of the squares, then we can easily make a infinite series by using the areas of the squares. Fortunately, I have got a pattern. We have to add the areas of the squares by using an interesting method. Let me describe it.

Look at the figure 2, at first, I have considered the area of the first square $(B D E F)$ as the first term of the infinite series. Then I have considered the sum of the areas of the second and third square (DGHI and $F J K L)$ as the second term of that infinite series. Then I have considered the sum of the areas of the $4^{\text {th }}, 5^{\text {th }}, 6^{\text {th }}$ and $7^{\text {th }}$ square $(I M N O, G P Q R, J S T U$ and $L V W X)$ as the third term of that infinite series. Similarly, I have considered the sum of the areas of the next eight squares as the fourth term of that infinite series. In this process I have continued. Now let me show the calculations.

Suppose $\mathrm{AB}=\mathrm{a}$ and $\mathrm{BC}=\mathrm{b}$. By the formula to calculate the length of one side of the inscribed square in a right triangle, we get the length of one side of the square $B D E F$

$=\frac{A B \cdot B C}{A B+B C}=\frac{a b}{a+b}$

So the area of the square $B D E F=\frac{a^{2} b^{2}}{(a+b)^{2}}$

Now the length of one side of the square $D G H I$ will be

$=\frac{E D \cdot D C}{E D+D C}=\frac{\frac{a b}{a+b} \times\left(b-\frac{a b}{a+b}\right)}{\frac{a b}{a+b}+\left(b-\frac{a b}{a+b}\right)}=\frac{a b^{3}}{(a+b)^{2}} \times \frac{a+b}{b(a+b)}=\frac{a b^{2}}{(a+b)^{2}}$

Again, the length of one side of the square $F J K L$ will be

$=\frac{A F \cdot F E}{A F+F E}=\frac{\left(a-\frac{a b}{a+b}\right) \times \frac{a b}{a+b}}{\left(a-\frac{a b}{a+b}\right)+\frac{a b}{a+b}}=\frac{a^{3} b}{(a+b)^{2}} \times \frac{a+b}{a(a+b)}=\frac{a^{2} b}{(a+b)^{2}}$

Now the sum of the areas of the squares will be

$=\left(\frac{a b^{2}}{(a+b)^{2}}\right)^{2}+\left(\frac{a^{2} b}{(a+b)^{2}}\right)^{2}=\frac{a^{2} b^{4}+a^{4} b^{2}}{(a+b)^{4}}=\frac{a^{2} b^{2}\left(a^{2}+b^{2}\right)}{(a+b)^{4}}$

Similarly, I will calculate the sum of the areas of the next four squares. Here I will show the calculations very shortly,

The length of one side of the square $I M N O$ will be

$=\frac{H I I I C}{H I+I C}=\frac{\frac{a b^{2}}{(a+b)^{2}} \times\left(b-\frac{a b}{a+b}-\frac{a b^{2}}{(a+b)^{2}}\right)}{\frac{a b^{2}}{(a+b)^{2}}+\left(b-\frac{a b}{a+b}-\frac{a b^{2}}{(a+b)^{2}}\right)}=\frac{a b^{3}}{(a+b)^{3}}$

Again, the length of one side of the square $G P Q R$ will be

$=\frac{E G \cdot G H}{E G+G H}=\frac{\left(\frac{a b}{a+b}-\frac{a b^{2}}{(a+b)^{2}}\right) \times \frac{a b^{2}}{(a+b)^{2}}}{\left(\frac{a b}{a+b}-\frac{a b^{2}}{(a+b)^{2}}\right)+\frac{a b^{2}}{(a+b)^{2}}}=\frac{a^{2} b^{2}}{(a+b)^{3}}$

Again, the length of one side of the square JSTU will be

$=\frac{K J \cdot J E}{K J+J E}=\frac{\frac{a^{2} b}{(a+b)^{2}} \times\left(\frac{a b}{a+b}-\frac{a^{2} b}{(a+b)^{2}}\right)}{\frac{a^{2} b}{(a+b)^{2}}+\left(\frac{a b}{a+b}-\frac{a^{2} b}{(a+b)^{2}}\right)}=\frac{a^{2} b^{2}}{(a+b)^{3}}$

Again, the length of one side of the square $L V W X$ will be

$=\frac{A L \cdot L K}{A L+L K}=\frac{\left(a-\frac{a b}{a+b}-\frac{a^{2} b}{(a+b)^{2}}\right) \times \frac{a^{2} b}{(a+b)^{2}}}{\left(a-\frac{a b}{a+b}-\frac{a^{2} b}{(a+b)^{2}}\right)+\frac{a^{2} b}{(a+b)^{2}}}=\frac{a^{3} b}{(a+b)^{3}}$

Now, the sum of the areas of the squares (IMNO, GPQR, JSTU and LVWX) will be 
$=\left(\frac{a b^{3}}{(a+b)^{3}}\right)^{2}+\left(\frac{a^{2} b^{2}}{(a+b)^{3}}\right)^{2}+\left(\frac{a^{2} b^{2}}{(a+b)^{3}}\right)^{2}+\left(\frac{a^{3} b}{(a+b)^{3}}\right)^{2}$

$=\frac{a^{2} b^{6}+a^{4} b^{4}+a^{4} b^{4}+a^{6} b^{2}}{(a+b)^{6}}$

$=\frac{a^{2} b^{2}\left\{\left(a^{2}+b^{2}\right)\left(a^{2}+b^{2}\right)\right\}}{(a+b)^{6}}$

$=\frac{a^{2} b^{2}\left(a^{2}+b^{2}\right)^{2}}{(a+b)^{6}}$

Now if we divide the area of the square $B D E F$ by the sum of the areas of the squares $D G H I$ and FJKL, we get

$=\frac{a^{2} b^{2}\left(a^{2}+b^{2}\right)}{(a+b)^{4}} \div \frac{a^{2} b^{2}}{(a+b)^{2}}=\frac{a^{2}+b^{2}}{(a+b)^{2}}$

Again, if we divide the sum of the areas of the squares $D G H I$ and $F J K L$ by the sum of the areas of the squares $I M N O, G P Q R, J S T U$ and $L V W X$, we get

$=\frac{a^{2} b^{2}\left(a^{2}+b^{2}\right)^{2}}{(a+b)^{6}} \div \frac{a^{2} b^{2}\left(a^{2}+b^{2}\right)}{(a+b)^{4}}=\frac{a^{2}+b^{2}}{(a+b)^{2}}$

Fortunately, both time the ratio is same. Now we get the beautiful pattern. If we multiply the sum of the areas of the squares IMNO, GPQR, JSTU and LVWX by $\frac{a^{2}+b^{2}}{(a+b)^{2}}$, we will get the sum of the areas of the next eight squares. So, now we can write,

The area of the right triangle $A B C$

$=$ area of $B D E F+($ area of $D G H I+$ area of $F J K L)+$

( area of $I M N O+$ area of $G P Q R+$ area of $J S T U+$ area of $L V W X)+\cdots$

$=\frac{a^{2} b^{2}}{(a+b)^{2}}+\frac{a^{2} b^{2}\left(a^{2}+b^{2}\right)}{(a+b)^{4}}+\frac{a^{2} b^{2}\left(a^{2}+b^{2}\right)^{2}}{(a+b)^{6}}+\cdots$

By this process we can present the area of any right triangle as the sum of an infinite series. Here the first term of the series, $a=\frac{a^{2} b^{2}}{(a+b)^{2}}$ and common ratio, $r=\frac{a^{2}+b^{2}}{(a+b)^{2}}$ which is less than $1(r<1)$. So, the series is convergent and the sum of this infinite series is

$=\frac{a}{1-r}=\frac{\frac{a^{2} b^{2}}{(a+b)^{2}}}{1-\frac{a^{2}+b^{2}}{(a+b)^{2}}}=\frac{a^{2} b^{2}}{(a+b)^{2}} \times \frac{(a+b)^{2}}{2 a b}=\frac{1}{2} a b$

$=\frac{1}{2} \times$ base $\times$ height

So we can get this formula from the infinite series. I think this is the difficult way to prove the formula! But the main motive of this article is not to prove the formula. I believe that this infinite series will show the beauty of mathematics. Students will get the message that one simple problem can be solved in many different interesting ways.

\section{Author's Biography}

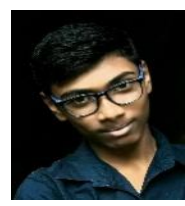

This is Salman Mahmud. Currently I am studying at BIAM Model School and College, Bogura, Bangladesh. My core research interest is in modern physics and pure mathematics. I like to spend time with my father (MD. Shah Alam) and my mother (MST. Sabina Yesmin). I also like to quarrel with my younger brother (Suyaib Sadik)!

Citation: Salman Mahmud, (2020). "Presenting the Area of a Right Triangle as the Sum of an Infinite Series”. International Journal of Scientific and Innovative Mathematical Research (IJSIMR), 8(3), pp.1-3. http://dx.doi.org/10.20431/2347-3142.0803001

Copyright: (C) 2020 Authors. This is an open-access article distributed under the terms of the Creative Commons Attribution License, which permits unrestricted use, distribution, and reproduction in any medium, provided the original author and source are credited. 